\title{
Acute and Chronic Cerebral White Matter Damage in Neonatal Hydrocephalus
}

\author{
Marc R. Del Bigio, Marcia C. da Silva, James M. Drake and Ursula I. Tuor
}

\begin{abstract}
The neonatal cat model of kaolin-induced hydrocephalus is associated with progressive and severe ventriculomegaly. In this experiment we studied the evolution of the histopathological changes in hydrocephalic $(n=23)$ cats from 5-168 days after the induction of hydrocephalus along with agematched controls $(n=10)$. In the periventricular white matter, extracellular edema and axonal damage were present within days of the onset of hydrocephalus. This was followed by reactive gliosis, white matter atrophy, and in some animals gross cavitation of the white matter. Even in the chronic, apparently compensated state there was ongoing glial cell death. Six cats were shunted an average of $23.6 \pm 6.5$ days after the induction of hydrocephalus because they were no longer able to feed independently. In spite of clinical improvement the white matter changes persisted. Overt cortical changes were minimal except where areas of white matter destruction encroached upon the deep layers. The white matter changes are very similar to those seen in periventricular leukomalacia and suggest that ischemia plays a role in neonatal brain injury caused by hydrocephalus.
\end{abstract}

\begin{abstract}
Résumé: Atteinte cérébrale aiguë et chronique de la substance blanche dans l'hydrocéphalie néonatale. Dans le modèle animal de l'hydrocéphalie induite par le kaolin chez le chat, on observe une ventriculomégalie progressive sévère. Nous avons étudié l'évolution des changements histopathologiques chez des chats hydrocéphalie $(n=23)$ et des contrôles appariés pour l'âge $(n=10)$, sur une période de 5 à 168 jours après l'induction de l'hydrocephalie. L'oedème extracellulaire et l'atteinte axonale au niveau de la substance blanche périventriculaire étaient présents dans les jours suivant le début de l'hydrocéphalie. Ces changements étaient suivis d'une gliose réactionnelle, d'atrophie de la substance blanche et, chez certains animaux, de la formation de cavités importantes dans la substance blanche. Même à l'état chronique apparemment compensé, le processus de mort cellulaire se poursuivait au niveau des cellules gliales. Six chats ont subi une dérivation, en moyenne $23.6 \pm 6.5$ jours après l'induction de l'hydrocéphalie, parce qu'ils ne pouvaient plus se nourrir. Les modifications de la substance blanche ont persisté malgré l'amélioration clinique. Les changements corticaux étaient minimes, sauf dans les régions où la destruction de la substance blanche empiétait sur les couches profondes. Les modifications de la substance blache sont très semblables à celles observées dans la leucomalacie périventriculaire, ce qui suggère que l'ischémie joue un rôle dans les lésions cérébrale néonatales causées par l'hydrocéphalie.
\end{abstract}

Can. J. Neurol. Sci. 1994; 21: 299-305

A review of the literature reveals that rapid onset of ventriculomegaly, especially in immature brains, leads to gross and microscopic white matter damage with less pronounced changes in the cerebral cortex.' The early neuropathological changes in the cerebral cortex of kittens with kaolin-induced hydrocephalus have been studied in detail by McAllister and coworkers. $^{2-6}$ Their methodical approach has allowed detection of subtle neuronal changes in several regions of the cortex. We have studied the pathophysiology of neonatal hydrocephalus using the same animal model and found that altered glucose utilization in the white matter correlates with a delay in myelination in the early stages of hydrocephalus and that the same areas exhibit reduced blood flow. These alterations can be corrected by early shunting of cerebrospinal fluid (CSF). We have also observed that the metabolic changes are minimal in the gray matter. ${ }^{7}$ It is unclear however, whether the cortical changes are a consequence of the white matter damage. Nor is it clear whether the structural white matter damage progresses in chronic hydro- cephalus. In this component of our experiments we examined the neuropathological changes caused by hydrocephalus of up to six months duration in cats. The cats were treated by a cerebrospinal fluid (CSF) shunting procedure only if they were unable to feed independently.

\section{Materials and Methods}

The experimental protocol was approved by the Animal Care Committee of The Hospital for Sick Children, Toronto, Canada.

From the Divisions of Neuropathology (M.R.D.B.), Neurosurgery (M.C.d.S., J.M.D.), and Neonatal Research (U.I.T.), the Hospital for Sick Children and University of Toronto, Toronto.

RECEIVED FEBRUARY 8, 1994. ACCEPTED IN FINAL FORM APRIL 26, 1994.

Reprint requests to: James M. Drake, MB FRCS, Division of Neurosurgery, Hospital for Sick Children, 555 University Avenue, Toronto, Ontario, Canada M5G IX8 
Thirty-one kittens, 5 to 10 days old obtained from a breeding colony established at The Hospital for Sick Children were randomly assigned either to littermate control or hydrocephalic groups. For induction of hydrocephalus the kittens were anesthetized with a mixture of $4 \%$ halothane and $50 \%$ nitrous oxide. The occipital scalp was shaved and washed, and the animals were positioned with maximal head flexion. A 25 gauge needle was inserted into the cisterna magna and a drop of CSF was allowed to drain before injection of $0.05 \mathrm{ml}$ of sterile distilled water (control group, $\mathrm{n}=10$ ) or $0.05 \mathrm{ml}$ of a sterile suspension of $25 \%$ kaolin (hydrocephalic group, $n=23$ ). The kittens were then returned to their mothers and examined daily. Their weight, anterior fontanel, gait development, and state of consciousness were assessed. Control cats were killed at selected intervals from 10-96 days of age. Fifteen hydrocephalic kittens not undergoing shunting were killed 5-168 days $(89.5 \pm 25.9$ days, mean \pm SEM) after the induction of hydrocephalus.

The six hydrocephalic kittens showing significant neurological deterioration required shunting 2-47 days (23.6 \pm 6.5 days, mean \pm SEM) after injection of kaolin. The decision to intervene was made if the cats were unable to feed independently and were failing to gain weight in parallel with their littermate controls. Before shunt insertion, the kittens were examined by magnetic resonance imaging to confirm ventriculomegaly. The kittens were anesthetized as described above and the occipital scalp, thorax, and abdomen were shaved and washed. Through a small occipital craniectomy and abdominal incision a ventriculoperitoneal shunt with a low-pressure distal slit valve was inserted. These cats were killed 16-179 days after shunting at 48-189 days of age.

The cats were killed under pentobarbital anesthesia by perfusion through the heart with $10 \%$ neutral buffered formalin. The brains were removed intact and placed in the same fixative for at least four days. All brains were cut to allow examination of three coronal sections of the cerebrum at the level of the caudate nucleus, the mid-thalamus including the hippocampi, and the posterior parietal/occipital lobes. Cross sections of the brainstem were made to allow examination of the midbrain, pons/cerebellum, medulla, and proximal cervical spinal cord. The tissues were dehydrated and embedded in paraffin. Tissue sections (5-6 $\mathrm{rm}$ thickness) were stained with Luxol fast blue / hematoxylin and eosin (LFB / HE). Selected sections were stained with HE alone or the Bielschowsky silver method. Using the peroxidaseantiperoxidase method, selected control and hydrocephalic brain sections were labeled with antibodies to glial fibrillary acidic protein (GFAP, polyclonal, 1/200 dilution, Dako), neurofilament $200 \mathrm{kD}$ (monoclonal, I/25, Sanbio), lysozyme (polyclonal, 1/200, Dako), or growth associated protein 43 (GAP-43, monoclonal, $1 / 200$, Boehringer-Mannheim), the latter using the antigen retrieval system (Biogenix, San Ramon, CA). The specimens were examined by one observer. Myelination was graded on an arbitrary semiquantitative scale of 0-4 (0-none; 1 patchy; 2 or 3-incomplete; 4-complete) on LFB/HE stained sections of the superior frontal gyrus, periventricular region, internal capsule, optic tract, middle cerebellar peduncle, medial lemniscus, and medullary pyramid. No attempt was made to grade other parameters beyond the presence of weak or strong staining.

\section{Results}

In all control cats the ventricles were slit-like (Figure 1). At 10-15 days, myelin staining was evident only in the optic tract, cerebellar peduncle, and medial lemniscus. By 28 days those sites exhibited grade 4 staining. Other regions exhibited grade 2 staining at 28 days and showed complete myelin staining intensity by 58 days, except for the superior frontal gyrus where staining was not complete until 67 days. In unmyelinated areas, axons stained only weakly using the silver stain and neurofilament antibody. GFAP immunoreactive myelination glia were evident in the white matter regions from the time that myelin staining was barely evident until the time that myelination was complete. In the mature cats, GFAP immunoreactivity was minimal and seen primarily in the subependymal region and surrounding blood vessels in the white matter. From the earliest age studied cortical lamination was well defined. At 10 days of age the parasagittal parietal cerebrum measured $5-6 \mathrm{~mm}$ from ventricle to pial surface and in the mature cats it measured $8-9 \mathrm{~mm}$. The cerebral cortex at all ages was approximately $2 \mathrm{~mm}$ in thickness.

The kaolin injected cats developed head enlargement with bulging of the anterior fontanel, separation of the skull sutures, downward deviation of the eyes, and were more clumsy than their littermate controls within 3 to 4 days. For approximately 1 week their weight gain lagged but thereafter it paralleled that of controls. Hydrocephalic kittens exhibited enlargement of the lateral and third ventricles and to a lesser degree the cerebral aqueduct and fourth ventricle (Figure 2). Only 1 of the 17 hydrocephalic cats had enlargement of the central canal of the spinal cord. Based on the compression of white matter and unfolding of the cerebral cortex, ventriculomegaly was assessed as slight to moderate for 5-13 days after the injection of kaolin. From 21-168 days after kaolin injection, ventriculomegaly ranged from moderate to severe with the parietal cerebrum

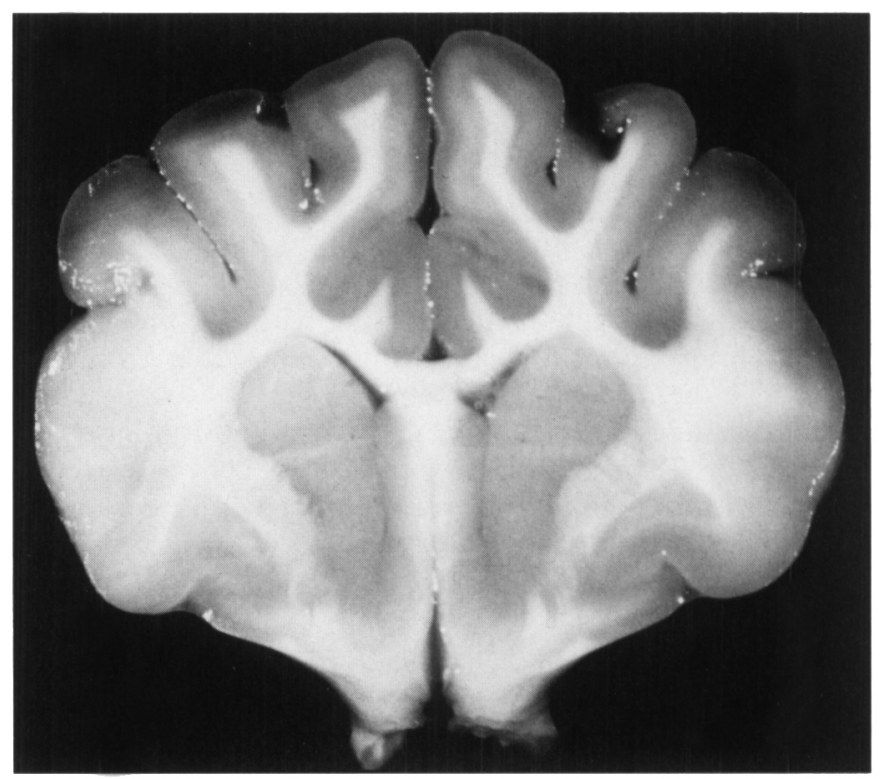

Figure 1: Photograph of a coronal section through the brain of a normal 48 day old cat showing the slit-like frontal horns of the lateral ventricles. 


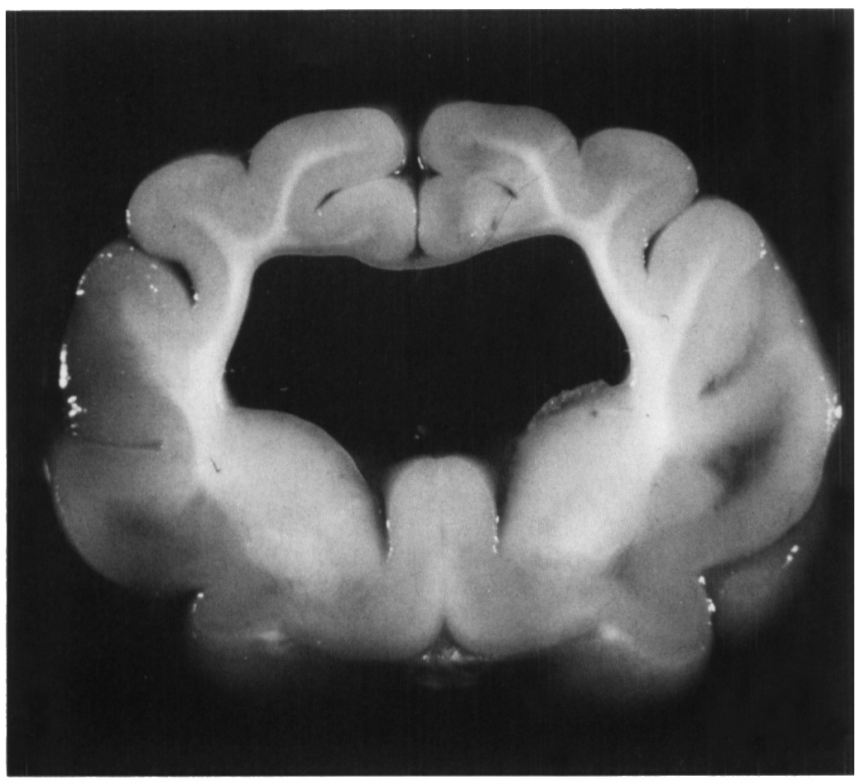

Figure 2: Photograph of a coronal section through the brain of a 58 day old cat 51 days after kaolin injection. The frontal horns are enlarged, the septum pellucidum is ruptured, and the white matter in the corpus callosum, periventricular region, and superior frontal gyrus is thinned. The degree of myelination in the white matter is reduced.

measuring from $1.5-5 \mathrm{~mm}$ thick. Most of the thinning was due to loss of white matter while the cortical thickness remained $1.5-2 \mathrm{~mm}$. The ventricular dilatation was most severe in the occipital lobe and dorsal aspects of the parietal and frontal lobes where the cerebrum was often focally thinned to less than $1 \mathrm{~mm}$. All of the cats with severe ventriculomegaly had a ruptured septum pellucidum and thin corpus callosum.

Microscopic examination revealed non-refractile particles of kaolin within macrophages in the subarachnoid space around the brainstem and adjacent to the basal forebrain. There was increased collagen staining in the leptomeninges in these areas but there were no abnormalities of the blood vessels passing through the inflammatory cell mass nor was there destruction or reactive gliosis in the adjacent brain. In four cats, kaolin-laden macrophages were identified in the third and lateral ventricles suggesting that they migrate from the cisterna magna region into the ventricles. Five to 13 days after injection of kaolin (7 cats) there was stretching and separation of the ependyma and a slight increase in GFAP immunoreactivity in the subependymal region. The unmyelinated axons and capillaries in the periventricular region and in the cores of gyri were dispersed in the enlarged extracellular space (Figure 3). From 9 to 13 days there were abundant swollen axons, some of which were in continuity, revealed by silver and neurofilament stains (Figure 4). There were also karyorrhectic cells and scattered foamy macrophages in the edematous periventricular white matter. Most of the GFAP immunoreactive cells in the white matter retained the morphologic characteristics of myelination glia (i.e., with immunoreactivity concentrated in one pole of the cell body) although some were obviously reactive astrocytes with long, coarse multipolar processes. Occasional mitotic figures were seen in the ependymal layer and among white matter glial cells. No abnormalities were detected in the internal capsule whose

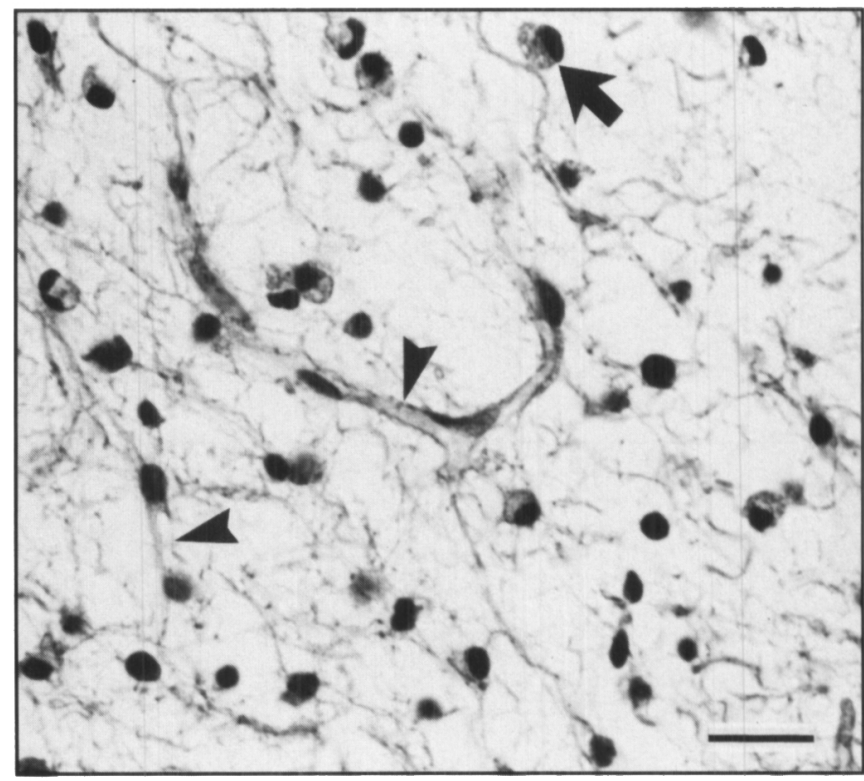

Figure 3: Light micrograph showing the edematous periventricular white matter with dispersed capillaries (arrowheads), axons, glial cells. and rare macrophages (arrow) in a cat that had been hydrocephalic for 21 days. $L F B / H E$ Bar $=20 \mu m$.

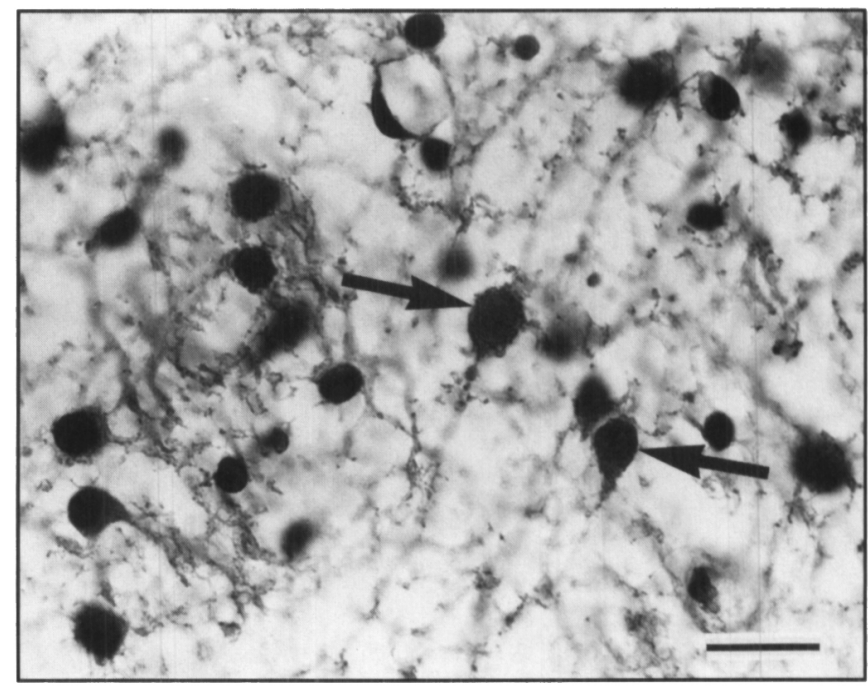

Figure 4. Light micrograph showing axonal swellings (arrows) in the periventricular white matter of a cat that had been hydrocephalic for 9 days. $\mathrm{HE}$ Bar $=20 \mu \mathrm{m}$.

degree of myelin staining was comparable to that in controls. The cerebral cortex and hippocampus were unremarkable with the exception of one cat in which reactive astrocytes were present in the deep cortex adjacent to the edematous white matter.

Twenty-one days after the induction of hydrocephalus the white matter remained edematous. Myelination was proceeding in the periventricular region, albeit more slowly than in controls of comparable age. Fifty-one days after the induction of hydrocephalus the periventricular white matter was thinned but there was no edema. Chains of oligodendrocytes and reactive astrocytes 
were sandwiched between narrow bundles of well myelinated axons (Figure 5). Scattered karyorrhectic glial cells were seen in the periventricular region and rarely in the internal capsule (Figure 6). None of these were GFAP immunoreactive. The perivascular spaces were often greatly enlarged (Figure 7). Silver staining and neurofilament immunoreactivity revealed moderate depletion of axons in the cores of gyri. The superior frontal gyrus of the three hydrocephalic cats older than 96 days had incomplete myelin staining (grade 2-3). In five of ten cats that had been hydrocephalic for 21-168 days there were non-

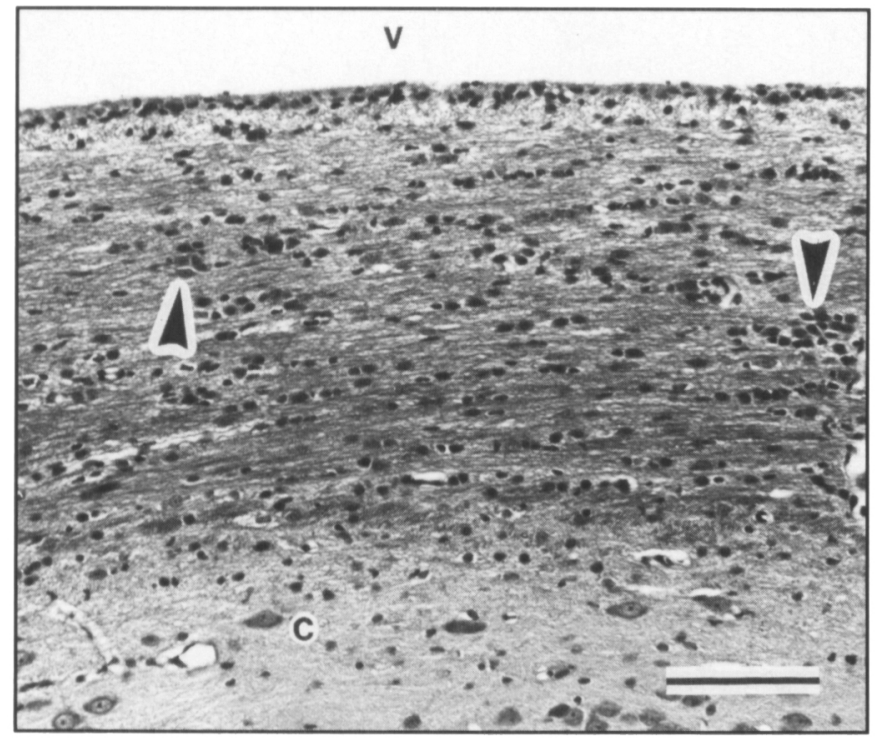

Figure 5: Light micrograph showing thinned periventricular white mat. ter in a cat that had been hydrocephalic for 51 days. The remaining axons are normally myelinated but their quantity is reduced, leaving glial cells packed in rows and clusters (arrowheads). The ependyma has been lost from the ventricle surface (v). $c=$ cortex. $L F B / H E$ Bar $=$ $100 \mu \mathrm{m}$.

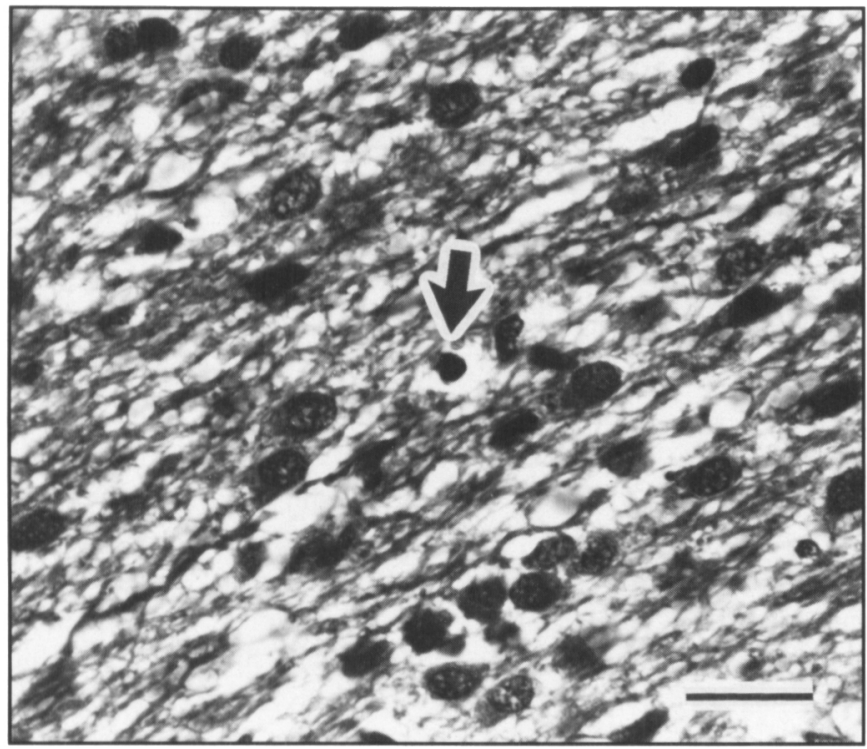

Figure 6: Higher magnification light micrograph of the area shown in Figure 5. A karyorrhectic glial cell nucleus (arrow) indicates that there is ongoing cell death. HE Bar $=20 \mu \mathrm{m}$. hemorrhagic often symmetric cavitations in the white matter. These varied in size, some involving only the core of a single gyrus others involving the majority of the periventricular region (Figure 8). Scattered small blood vessels and a loose web of reactive astrocytes traversed the smaller cavities. Adjacent to these cavities myelin debris, swollen axons, and cells with clumped chromatin suggestive of cell death could be identified in animals of 28 and 34 days of age. At 58 days there were macrophages in the periphery of the cavities and at 168 days only reactive astrocytes surrounded the cavities (Figure 9). In the caudate nucleus and thalamus of two cats there was a slight increase in cell density due to the presence of reactive astroglia. In cats with severe white matter destruction and ventriculomegaly the deep cortical layers were distorted, causing the neurons to have an elongated profile. In one cat the deep parietal cortex was focally destroyed where there was complete loss of the underlying white matter (Figure 10). In general the extent of white matter damage and associated gray matter changes correlated with the severity of ventriculomegaly rather than the duration

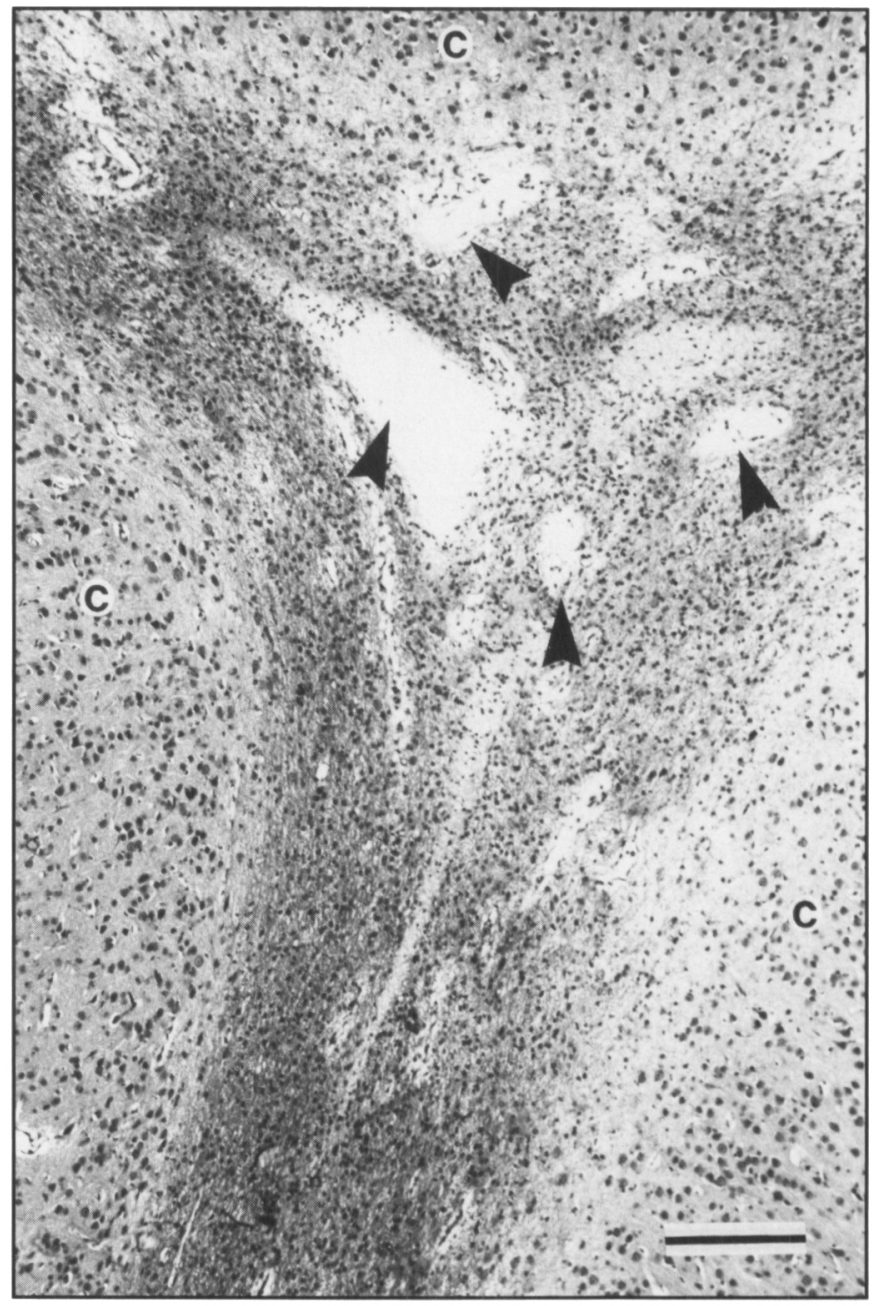

Figure 7: Light micrograph showing the white matter core of the superior parietal gyrus in a cat that had been hydrocephalic for 51 days. The edema has resolved but, indicative of atrophy, the perivascular spaces are greatly enlarged (arrowheads). $c=$ cortex. LFB $/ H E$ Bar $=200 \mu \mathrm{m}$. 


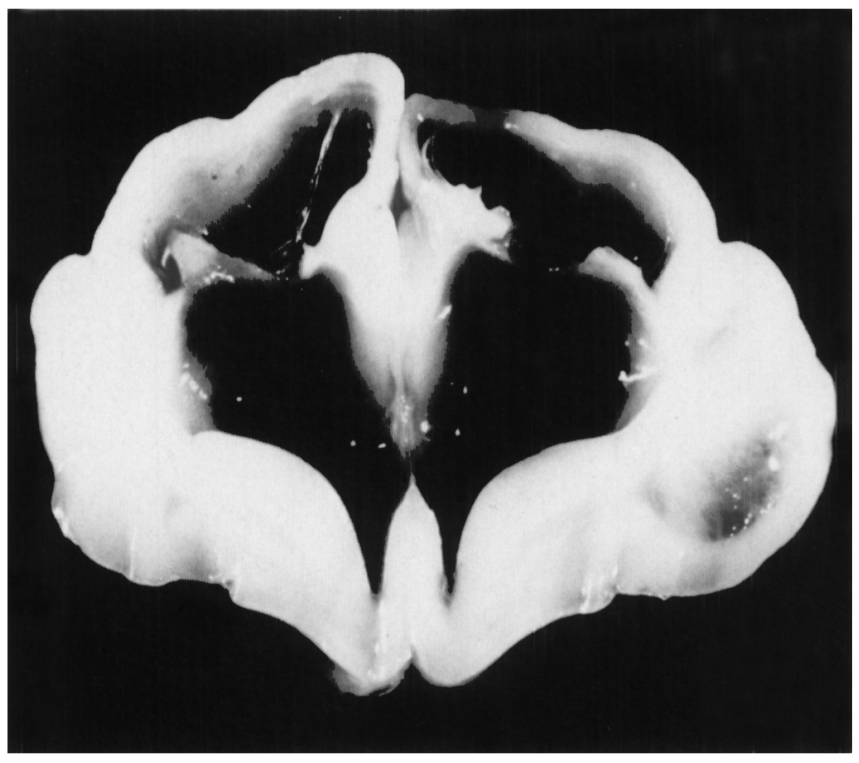

Figure 8: Photograph of a coronal section of the brain of a cat which had been hydrocephalic for 21 days. There is cavitation of the periventricular white matter with thinning of the cerebral cortex.

of hydrocephalus. No changes other then mild flattening were identified in the hippocampus at any stage.

Because of severe neurological impairment six cats required shunting $2,11,24,25,33$, and 47 days after the injection of kaolin. Following shunting they were once again able to feed independently. At age 48-189 days when they were killed, all had slight to moderate dilatation of the ventricles despite apparent functioning of the shunt. One cat had a dilated central canal. In all shunted cats the ventricle surface was only focally covered by ependyma although ependymal cells forming rosettes were buried in the gliotic subependymal layer. One cat had inflammation indicative of ventriculitis as well as cavitation of the white matter. None of the other five cats, despite being allowed to reach maturity, had normal periventricular white matter. The internal capsule always had reduced myelin staining (grade 2-3). The superior frontal and parietal gyri had a reduced population of axons (Figure 11) and the white matter was gliotic. Two of the five cats had cavitation of the white matter with scattered hemosiderin-laden macrophages. The bleeding was probably caused by insertion of the shunt which passed through the cavity. The cerebrum thickness was $1.5-7 \mathrm{~mm}$. In rare areas, where there was full thickness destruction of adjacent white matter, the cortex was thin and lacked a distinct laminar pattern. In two cats the hippocampus adjacent to the ventricle was flattened and contained rare reactive astrocytes. There were no abnormalities seen in the basal ganglia, choroid plexus, cerebellum, or brainstem except for reduced intensity of myelin staining in the medullary pyramids (grade 3 ). The magnitude of residual white matter damage was comparable to that in some of the hydrocephalic cats which did not require a shunt. However, ongoing cell death was never identified in the periventricular white matter of shunted cats.

Staining of the brains for GAP-43 was disappointing in that there were no major changes in hydrocephalic animals. At 13 days the basal ganglia were most intensely stained followed by

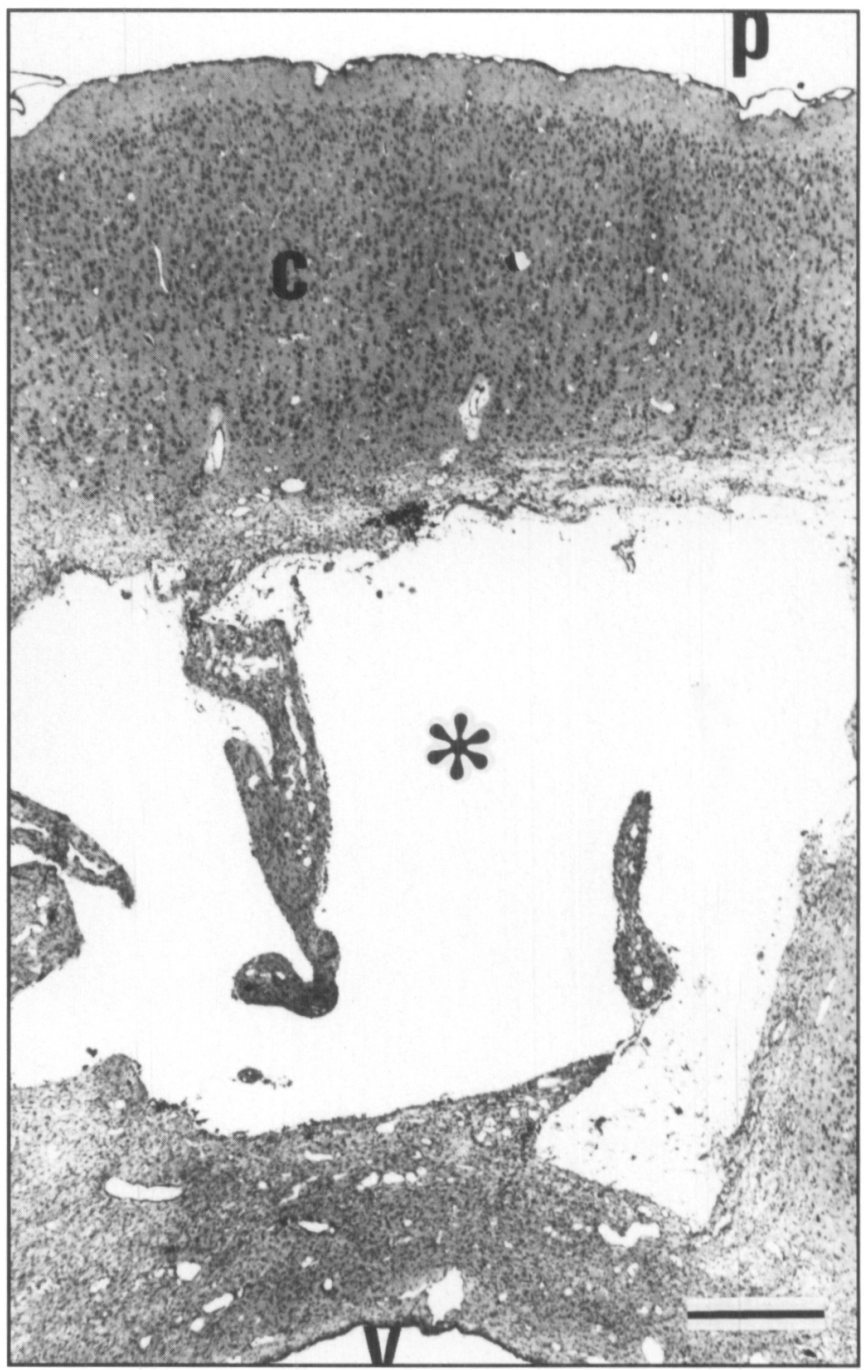

Figure 9: Light micrograph showing periventricular cavitation (*) in the brain shown in Figure 8. The subcortical white matter is destroyed and the cyst is bridged by bands of reactive astrocytes. $p=$ pial surface. $c=$ cortex,$v=$ ventricle. $L F B / H E \quad B a r=500 \mu m$.

deep cortical neuropil, white matter, and superficial cortical layers. At 28 days the molecular layer of the cerebrum showed the most intense staining and white matter the least. At 48 days the cortex and basal ganglia were less intensely stained than at younger ages and white matter showed minimal staining. At 96 days the overall intensity was further decreased. The pattern of gray matter immunoreactivity was unchanged by the presence of hydrocephalus. However, in the edematous white matter there was intense staining of scattered stellate cells. On adjacent sections stained for GFAP some of the corresponding cells were immunoreactive.

\section{Discussion}

This experimental model of neonatal hydrocephalus displays severe and progressive enlargement of the lateral ventricles. At the time of kaolin injection the maturity of the cat brain corresponds to that of a premature human infant. ${ }^{8}$ As in human neonates, the rate of dilatation is variable and the severity of 


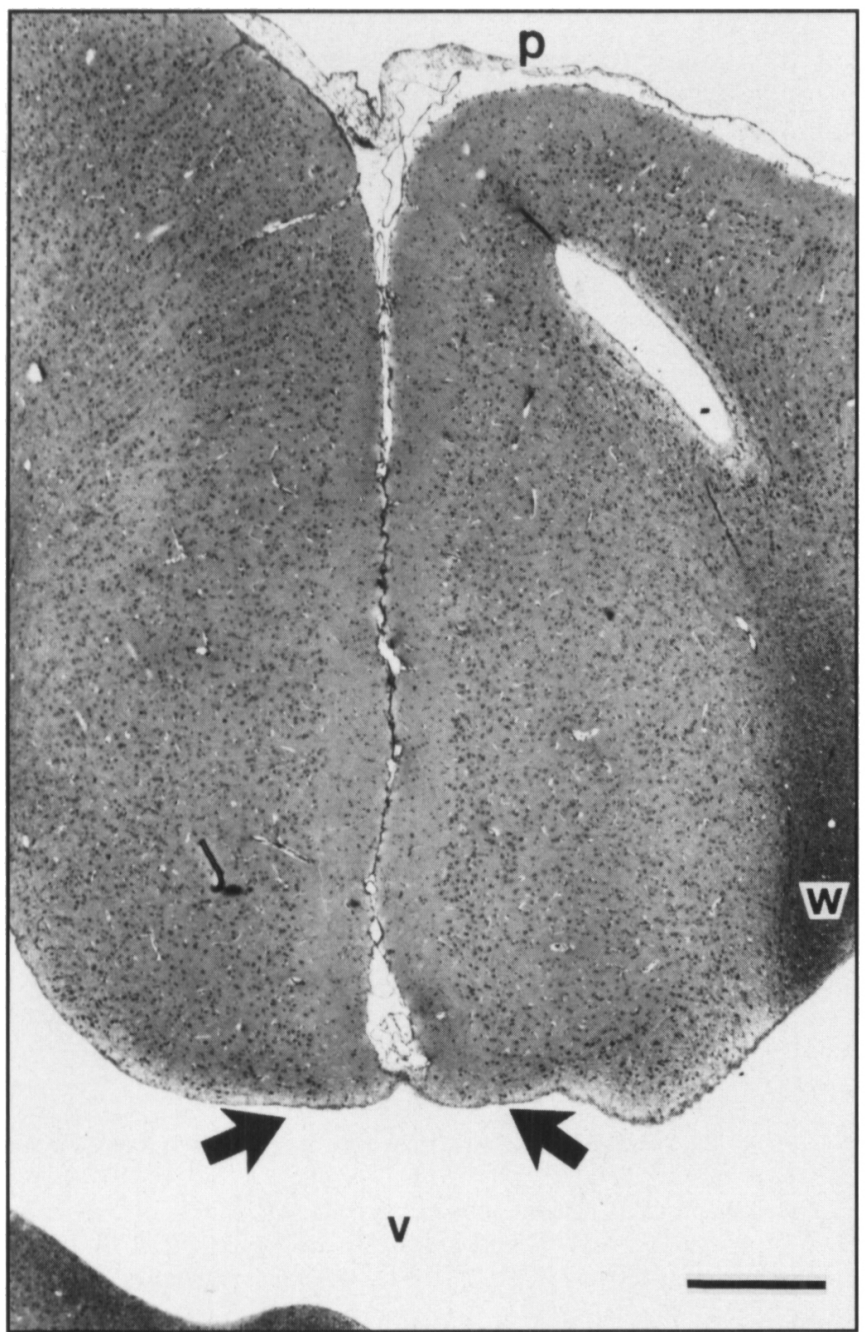

Figure 10: Light micrograph showing the lateral frontal cortex of a cat that had been hydrocephalic for 168 days. White matter is absent and the cerebral cortex is lost at the depth of the sulcus (arrows). In other areas the white matter $(w)$ is well myelinated. $p=$ pial surface, $v=v e n$ tricle. $L F B / H E$ Bar $=500 \mu m$.

clinical symptoms does not necessarily correlate with size of the ventricles. Also as in human neonates, the ventricles do not necessarily return to normal size promptly after shunting. ${ }^{9}$ The particularly destructive nature of hydrocephalus in this model is likely due to the relatively acute obstruction to CSF flow. Whether damage occurs this quickly in human brains is unclear. Previous reports concerning animal models that exhibit such severe white matter damage have concentrated on the short-term cortical changes, with and without shunting.' The long-term pathological changes in the white matter of the neonatal cat model are evident using classical neuropathological methods. Enlargement of the extracellular compartment in the cerebral white matter occurs within a few days and is probably due to impaired flow of extracellular fluid from the parenchyma into the ventricles.' During the same interval axonal damage occurs, as has been previously demonstrated in animals ${ }^{10-13}$ and

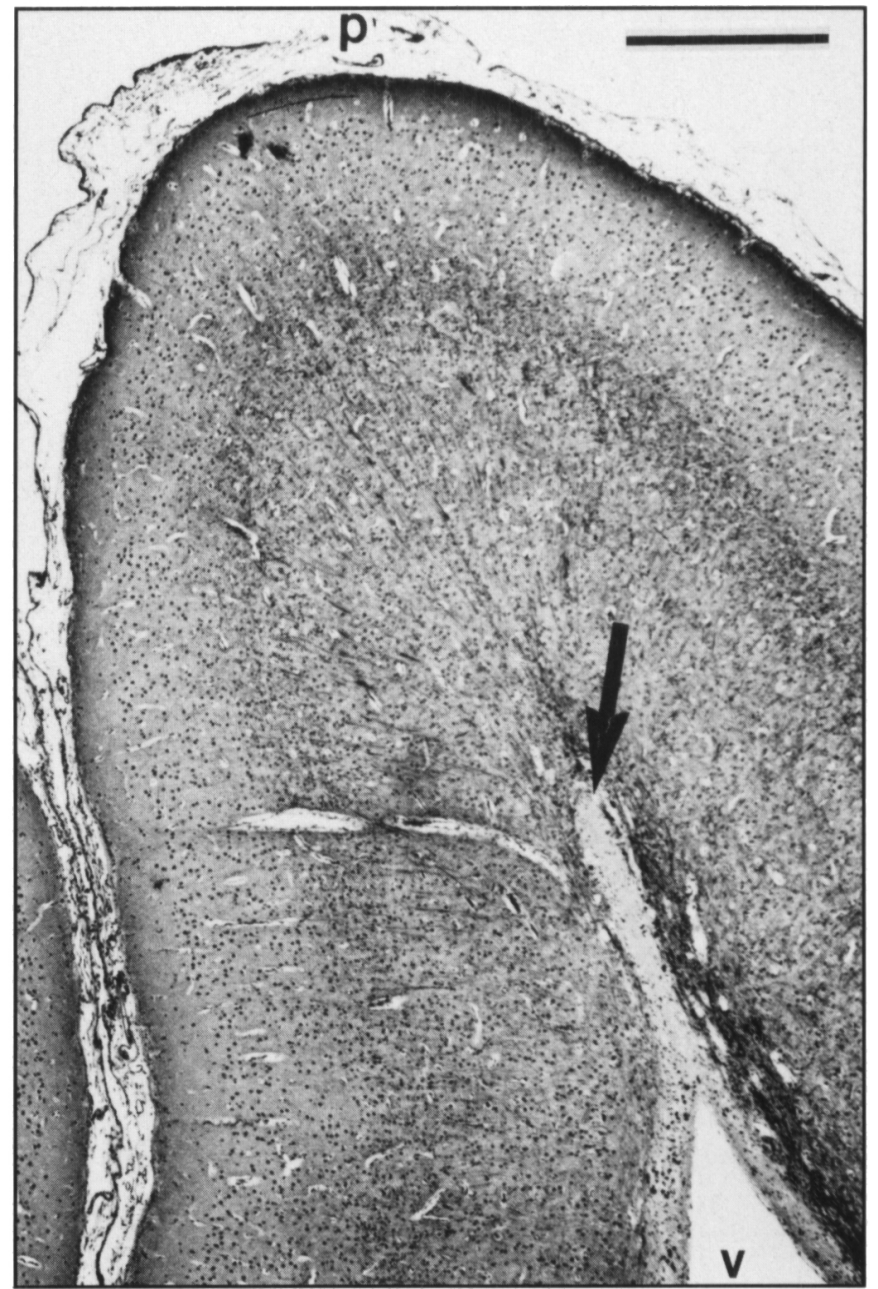

Figure 11: Light micrograph showing the superior parietal gyrus of a cat that had been hydrocephalic for 2 days after which a shunt was placed on the contralateral side. There was successful reduction of the ventricle size for 179 days. The ventricle lining is gliotic and only a few axons (stained black, arrow) remain in the core of the gyrus. A gliotic band (pale staining, arrow) extends from the ventricle (v) to the deep cortical layer. $p=$ pial surface. Bielschowsky silver method. Bar $=500$ $\mu m$.

humans. ${ }^{14}$ The pathogenesis of this change remains unclear but experimental models of head injury suggest that axonal swelling may be the result of distortion to the axonal cytoskeleton or altered permeability of the axolemma rather than an acute disruption of axon continuity. ${ }^{15.16}$ In kittens that have been hydrocephalic for three weeks there are regions of anaerobic glycolysis in the periventricular white matter, generally decreased blood flow in the white matter, and normal metabolism in the gray matter. ${ }^{7}$ A combination of slow physical distortion and ischemia likely contributes to the axonal injury in hydrocephalus .' The ensuing tissue response is one of astroglial proliferation and hypertrophy. ${ }^{17}$ While GAP-43 is usually associated with axonal growth, ${ }^{18}$ we observed increased staining only in the white matter and no changes in gray matter. Recently it was shown that GAP-43 and myelin are reciprocally expressed in white matter axons. ${ }^{19}$ Our observations could 
reflect proliferation of glial cells in the white matter because GAP-43 has been previously reported in immature oligodendrocytes in brain and astrocytes in early cultures. ${ }^{20.21}$ Nevertheless, we cannot exclude the possibility of insensitivity in our methods and we suggest that quantitative Western blots of tissue homogenates would be a more sensitive method to detect diffuse changes in the gray and white matter.

As edema resolves and white matter density returns to normal, the axonal loss becomes apparent with loss of silver staining and neurofilament immunoreactivity, loss of myelin staining, and gross tissue atrophy. In severely affected animals the tissue destruction results in cystic areas surrounded by reactive astrocytes and enlarged perivascular spaces. In the same experimental model the cortical changes include thinning, edema in the deep layers, alterations to the nuclei of neurons, and changes to neuronal dendrites which do not recover even after successful shunting. ${ }^{2-6}$ The coexistence of white matter destruction and only relatively subtle cortical changes suggests that the neuronal changes may be largely secondary to the retrograde effects of axonal damage and loss of axodendritic contacts due to interruption of short and long association fibers and commissural fibers. The apparent delay in myelination is the result of two factors. First, there is loss of axons which would otherwise become myelinated. Second, the general metabolic compromise and glial cell death in the periventricular white matter indicate a possible impairment of oligodendrocyte function. ${ }^{7.22}$ Dying glial cells could also be astroglial daughter cells which are known in some circumstances to die shortly after mitosis. ${ }^{23}$ The cystic white matter changes along with the ongoing death of periventricular glial cells are almost identical to the changes observed in periventricular leukomalacia, a lesion known to be hypoxic-ischemic in nature. ${ }^{24}$ These observations support data which indicate that the brain injury due to hydrocephalus is contributed to by hypoxia-ischemia. ${ }^{\prime}$ As is the case with other destructive lesions, the white matter damage in prolonged hydrocephalus is not reversible by shunting of CSF and emphasizes the need for early intervention.

\section{ACKNOWLEDGEMENTS}

This study was supported by grants from the Physicians Services Incorporated Foundation and the Appugliesi Fund. S. Bascaramurty and B. Biederman assisted with the animal care. I. Diplock, M. Ho, and C. Dempniak processed the tissues for histological study.

\section{REFERENCES}

1. Del Bigio MR. Neuropathological changes caused by hydrocephalus. Acta Neuropathol 1993; 85: 573-585.

2. Hale PM, McAllister JP, Katz SD, et al. Improvement of cortical morphology in infantile hydrocephalic animals after ventriculoperitoneal shunt placement. Neurosurgery 1992; 31: 10851096.

3. Kriebel RM, Shah AB, McAllister JP. The microstructure of cortical neuropil before and after decompression in experimental infantile hydrocephalus. Exp Neurol 1993; 119: 89-98.

4. Lovely TJ, McAllistar JP, Miller DW, Lamperti AA, Wolfson BJ. Effects of hydrocephalus and surgical decompression on cortical norepinephrine levels in neonatal cats. Neurosurgery 1989;24: 43-52.

5. McAllister JP, Cohen MI, O'Mara KA, Johnson MH. Progression of experimental infantile hydrocephalus and effects on ventriculoperitoneal shunts: an analysis correlating magnetic resonance imaging with gross morphology. Neurosurgery 1991; 29: 329340.

6. Wright LC, McAllister JP, Katz SD, et al. Cytological and cytoarchitectural changes in the feline cerebral cortex during experimental infantile hydrocephalus. Pediatr Neurosurg 1991; 16: 139-155.

7. Chumas PD, Drake JM, Del Bigio MR, Da Silva M, Tuor Ul. Anaerobic glycolysis preceding white-matter destruction in experimental neonatal hydrocephalus. J Neurosurg 1994; 80: $491-501$

8. Chugani HT, Hovda DA, Villablanca JR, Phelps ME. Metabolic maturation of the brain: a study of local cerebral glucose utilization in the developing cat. J Cereb Blood Flow Metabol 1991; 11: $35-47$

9. Cardoso ER, Del Bigio MR. Age-related changes of cerebral ventricular size. Part II: Normalization of ventricular size following shunting. Acta Neurochir 1989; 97: 135-138.

10. De SN. A study of the changes in the brain in experimental internal hydrocephalus. J Pathol Bacteriol 1950; 62: 197-208.

11. Rubin RC, Hochwald GM, Tiell M, Mizutani H, Ghatak N. Hydrocephalus: 1. Histological and ultrastructural changes in the pre-shunted cortical mantle. Surg Neurol 1976; 5: 109-114.

12. Weller RO, Wisniewski H, Shulman K, Terry RD. Experimental hydrocephalus in young dogs: histological and ultrastructural study of brain tissue damage. J Neuropathol Exp Neurol 1971: 30: 613-626.

13. Yamada H, Yokota A, Furuta A, Horie A. Reconstitution of shunted mantle in experimental hydrocephalus. J Neurosurg 1992; 76: 856-862.

14. Weller RO, Williams BN. Cerebral biopsy and assessment of brain damage in hydrocephalus. Arch Dis Child 1975; 50: 763-768.

15. Povlishock JT. Traumatically induced axonal injury: pathogenesis and pathobiological implications. Brain Pathol 1992; 2: 1-12.

16. Schweitzer JB, Dohan FC. Diffuse axonal injury: windows for therapeutic intervention allowed by its pathobiology. Virchows Arch A 1993; 423: 153-156.

17. Del Bigio MR, Bruni JE. Periventricular pathology in hydrocephalic rabbits before and after shunting. Acta Neuropathol 1988; 77 : 186-195.

18. Strittmatter SM, Vartanian T, Fishman MC. GAP-43 as a plasticity protein in neuronal form and repair. J Neurobiol 1992; 23: 507520.

19. Kapfhammer JP, Schwab ME. Inverse patterns of myelination and GAP-43 expression in the adult CNS: neurite growth inhibitors as regulators of neuronal plasticity? J Comp Neurol 1994; 340: 194-206.

20. Curtis R, Hardy R, Reynolds R, Spruce BA, Wilkin GP. Down-regulation of GAP-43 during oligodendrocyte development and lack of expression by astrocytes in vivo: implications for macroglial differentiation. Eur J Neurosci 1991; 3: 876-886.

21. Vitkovic L, Mersel M. Growth-associated protein 43 is down-regulated in cultured astrocytes. Metabolic Brain Dis 1989; 4: 47-53.

22. Davison AN, Dobbing J. Myelination as a vulnerable period in brain development. Brit Med Bull 1966; 22: 40-44.

23. Korr H. Proliferation and cell cycle parameters of astrocytes. In: Federoff S, Vernadakis A, eds. Astrocytes: Cell Biology and Pathology of Astrocytes. Volume 3. Orlando: Academic Press, 1986: 77-127

24. Gilles FH, Murphy SF. Perinatal telencephalic leucoencephalopathy. J Neurol Neurosurg Psychiatry 1969; 32: 404-413. 